Louisiana State University

LSU Digital Commons

Faculty Publications

Department of Chemistry

$12-14-2019$

\title{
Monitoring the growth dynamics of colloidal gold-silver core-shell nanoparticles using in situ second harmonic generation and extinction spectroscopy
}

\author{
Jeewan C. Ranasinghe \\ Louisiana State University \\ Asela S. Dikkumbura \\ Louisiana State University \\ Prakash Hamal \\ Louisiana State University \\ Min Chen \\ Louisiana State University \\ Rami A. Khoury \\ Louisiana State University
}

See next page for additional authors

Follow this and additional works at: https://digitalcommons.Isu.edu/chemistry_pubs

\section{Recommended Citation}

Ranasinghe, J., Dikkumbura, A., Hamal, P., Chen, M., Khoury, R., Smith, H., Lopata, K., \& Haber, L. (2019). Monitoring the growth dynamics of colloidal gold-silver core-shell nanoparticles using in situ second harmonic generation and extinction spectroscopy. Journal of Chemical Physics, 151 (22) https://doi.org/ $10.1063 / 1.5127941$

This Article is brought to you for free and open access by the Department of Chemistry at LSU Digital Commons. It has been accepted for inclusion in Faculty Publications by an authorized administrator of LSU Digital Commons. For more information, please contact ir@lsu.edu. 


\section{Authors}

Jeewan C. Ranasinghe, Asela S. Dikkumbura, Prakash Hamal, Min Chen, Rami A. Khoury, Holden T. Smith, Kenneth Lopata, and Louis H. Haber 


\section{Monitoring the growth dynamics of colloidal gold-silver core-shell nanoparticles using in situ second harmonic generation and extinction spectroscopy}

Cite as: J. Chem. Phys. 151, 224701 (2019); https://doi.org/10.1063/1.5127941

Submitted: 16 September 2019 . Accepted: 15 November 2019. Published Online: 09 December 2019

Jeewan C. Ranasinghe, Asela S. Dikkumbura, Prakash Hamal, Min Chen, Rami A. Khoury, Holden T. Smith, (D) Kenneth Lopata, (D) Louis H. Haber, et al.

\section{COLLECTIONS}

EP This paper was selected as an Editor's Pick
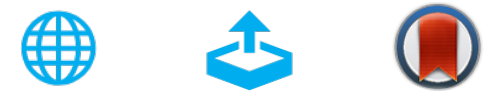

\section{ARTICLES YOU MAY BE INTERESTED IN}

Enhancement of the second harmonic signal of nonlinear crystals by self-assembled gold nanoparticles

The Journal of Chemical Physics 152, 104711 (2020); https://doi.org/10.1063/1.5139893

Suppression of dynamic disorder in fullerenes at metal-organic interfaces

The Journal of Chemical Physics 151, 214706 (2019); https://doi.org/10.1063/1.5123739

Phonon dispersion in two-dimensional solids from atomic probability distributions

The Journal of Chemical Physics 151, 224107 (2019); https://doi.org/10.1063/1.5132419

Challenge us.

What are your needs for periodic signal detection?

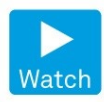

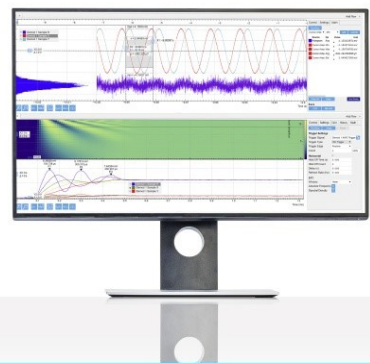

Zurich

Instruments 


\title{
Monitoring the growth dynamics of colloidal gold-silver core-shell nanoparticles using in situ second harmonic generation and extinction spectroscopy
}

\author{
Cite as: J. Chem. Phys. 151, 224701 (2019); doi: 10.1063/1.5127941 \\ Submitted: 16 September 2019 - Accepted: 15 November 2019 • \\ Published Online: 9 December 2019
}

Jeewan C. Ranasinghe, Asela S. Dikkumbura, Prakash Hamal, Min Chen, Rami A. Khoury, Holden T. Smith, Kenneth Lopata, and Louis H. Haber ${ }^{\text {a) }}$

\author{
AFFILIATIONS \\ Department of Chemistry, Louisiana State University, Baton Rouge, Louisiana 70803, USA
}

author to whom correspondence should be addressed: Ihaber@lsu.edu

\begin{abstract}
The growth dynamics of gold-silver core-shell (Au@Ag) nanoparticles are studied using in situ time-dependent second harmonic generation (SHG) and extinction spectroscopy to investigate the nanoparticle shell formation. The silver shell is grown by reduction of silver cations onto a $14 \mathrm{~nm}$ gold core using ascorbic acid in colloidal aqueous solution under varying reaction concentrations producing Au@Ag nanoparticles of final sizes ranging from 51 to $78 \mathrm{~nm}$ in diameter. The in situ extinction spectra show a rapid increase in intensity on the timescale of 5-6 s with blue shifting and narrowing of the plasmonic peak during the silver shell formation. The in situ SHG signals show an abrupt rise at early times of the reaction, followed by a time-dependent biexponential decrease, where the faster SHG lifetime corresponds to the timescale of the shell growth, and where the slower SHG lifetime is attributed to changes in the nanoparticle surface charge density. A large enhancement in the SHG signal at early stages of the reaction is caused by plasmonic hot spots due to the nanoparticle surface morphology, which becomes smoother as the reaction proceeds. The final extinction spectra are compared to finite-difference time-domain (FDTD) calculations, showing general agreement with experiment, where the plasmon peak red shifts and increases in spectral width as the silver shell thickness increases. These in situ SHG and extinction spectroscopy results, combined with FDTD calculations, help characterize the complicated processes involved in colloidal nanoparticle shell formation in real time for developing potential plasmon-enhanced nanomaterial applications.
\end{abstract}

Published under license by AIP Publishing. https://doi.org/10.1063/1.5127941

\section{INTRODUCTION}

The study of plasmonic nanoparticles is a field of intense research due to their potential applications in nanomedicine, photothermal therapy, molecular sensing, catalysis, and photovoltaics. $^{1-14}$ Gold and silver nanoparticles have localized surface plasmon resonances (LSPRs), which are characterized by the coherent oscillations of free electrons at the nanoparticle surface caused by incident illumination, leading to enhanced optical absorption and scattering processes. ${ }^{15}$ Bimetallic nanoparticles in core-shell configurations have emerged as a new class of plasmonic nanoparticles due to the improved optoelectronic tunability by adjusting their overall size, shape, and composition, as well as their individual core and shell sizes. ${ }^{16-18}$ Core-shell architectures composed of gold and silver are especially attractive for nanomedicine, photothermal therapy, and noninvasive bioimaging as a consequence of their biocompatibility and large plasmonic response. ${ }^{12,19-21}$ For further development of core-shell nanoparticle applications, a detailed understanding of the mechanisms and kinetics of nanoparticle shell formation is needed, which can help provide new approaches in tailoring the chemical, structural, and plasmonic properties for the desired nanomaterial technologies.

Several methods have been developed for the synthesis of gold-silver core-shell nanoparticles including procedures that rely 
on chemical, ${ }^{12,22-25}$ microwave-assisted, ${ }^{26}$ photochemical, ${ }^{27,28}$ and sonochemical routes. ${ }^{29}$ In chemical synthesis strategies, $\mathrm{Ag}^{+}$is typically reduced on the surface of a colloidal core gold nanoparticle for independent control of both the gold core size and the silver shell thickness. The usage of a mild reducing agent, such as ascorbic acid (AA), facilitates the reduction of $\mathrm{Ag}^{+}$selectively onto the colloidal gold nanoparticle surface while preventing the formation of free silver nanoparticles. ${ }^{12,18}$ Analytical techniques such as transmission electron microscopy (TEM), scanning electron microscopy, atomic force microscopy, dynamic light scattering, and extinction spectroscopy are important tools for the ex situ characterization of core-shell nanoparticles after the synthesis is complete. $8,12,13,17,30$

In order to investigate the growth dynamics of nanomaterials in real time, in situ characterization techniques have been developed that perform time-dependent measurements as the nanoparticle formation occurs under varying reaction conditions. For example, the nucleation and growth of silver nanoparticles in aqueous solution was studied under varying electron beam currents using in situ scanning transmission electron microscopy. ${ }^{31}$ Additionally, in situ TEM was used to investigate different pathways of silver shell growth around a cubic gold nanoparticle core in aqueous solution using the reducing agent AA with different capping agent concentrations. ${ }^{32}$ The nucleation and growth of silver nanoparticles by sodium borohydride reduction in water was studied using in situ extinction spectroscopy combined with in situ small angle X-ray scattering. ${ }^{33}$ The formation of gold nanoshells onto silica nanoparticles was investigated using in situ extinction spectroscopy combined with in situ second harmonic scattering measurements. ${ }^{34}$ In our recent work, we studied the seedmediated growth dynamics of colloidal gold nanoparticles under chemical reduction using in situ second harmonic generation (SHG) and in situ extinction spectroscopy. ${ }^{35}$ These different in situ techniques provide key insights into nanoparticle synthesis reactions and core-shell growth processes, leading to improved nanoscale engineering.

Second harmonic generation is a surface-sensitive nonlinear optical technique in which two photons of frequency $\omega$ interact coherently to generate a photon with twice the frequency at $2 \omega .^{36-41}$ For centrosymmetric materials and bulk solutions, SHG only occurs at an interface where the inversion symmetry is broken. Therefore, SHG spectroscopy is a powerful method for investigating the colloidal nanoparticle surface and the corresponding surface chemistry during in situ nanoparticle growth reactions. The SHG electric field $E_{S H G}$ originates from the second-order and third-order nonlinear susceptibilities, $\chi^{(2)}$ and $\chi^{(3)}$, respectively, with

$$
E_{S H G}=\sqrt{I_{S H G}}=\chi^{(2)} E_{\omega} E_{\omega}+\chi^{(3)} E_{\omega} E_{\omega} \Phi_{0} \cos \varphi e^{i \varphi},
$$

where $E_{\omega}$ is the incident optical electric field at frequency $\omega, \Phi_{0}$ is the electrostatic potential at the nanoparticle surface, $\varphi$ is the phase angle of the $\chi^{(3)}$ term, and $I_{S H G}$ is the SHG intensity. ${ }^{42-44}$ The $\chi^{(2)}$ term for SHG is due to the two-photon resonant and nonresonant spectroscopy of the sample, while the $\chi^{(3)}$ term for SHG arises from the nanoparticle electrostatic surface potential causing a net polarization of the bulk solvent. In the case of small $\varphi, E_{S H G} \propto \chi^{(2)}+\chi^{(3)} \Phi_{0}$. Previous work on SHG has investigated a large variety of samples including biological interfaces, ${ }^{45-48}$ interfacial acid-base chemistry, ${ }^{49}$ surface potential measurements, ${ }^{4,50} \mathrm{TiO}_{2}$ microparticles, ${ }^{51}$ and metallic, plasmonic nanoparticles composed of gold, silver, and gold-silver alloys. ${ }^{52-58}$ Our recent study of seed-mediated growth dynamics of colloidal gold nanoparticles using in situ SHG spectroscopy demonstrated a size-dependent nanoparticle growth lifetime, which is dominated by plasmonic hot spots and a surface morphology that becomes smoother and more uniform as a function of reaction time.

In this article, in situ SHG combined with in situ extinction spectroscopy is used to study the growth dynamics of colloidal goldsilver core-shell nanoparticles. The silver shells are grown by reduction of $\mathrm{Ag}^{+}$onto $14 \mathrm{~nm}$ gold seed nanoparticles using AA in water under three different reaction concentrations to produce Au@Ag nanoparticles of final sizes ranging from 51 to $78 \mathrm{~nm}$ in diameter. The time-dependent SHG signals and extinction spectra are analyzed to elucidate the mechanisms of the silver shell growth process and to determine the characteristic nanoparticle growth lifetimes as a function of the shell thickness. The extinction spectroscopy results show that the nanoparticle growth lifetime increases as the shell thickness increases. Additionally, the in situ SHG time traces exhibit biexponential behavior where the faster lifetimes are in excellent agreement with the lifetimes obtained from the extinction measurements and the slower lifetimes are attributed to additional surface reactions that change the nanoparticle surface charge densities. The in situ results also display large SHG enhancements at early stages in the shell growth process caused by plasmonic hot spots from rough, uneven nanoparticle surfaces that become smoother and more uniform as the reaction proceeds. The final extinction spectra are compared with finite-difference time-domain (FDTD) calculations, showing general agreement with the plasmon peak red shifting and increasing in spectral width as the silver shell thickness increases. The in situ SHG and extinction spectroscopy measurements provide valuable information regarding colloidal nanoparticle coreshell growth processes for developing controlled plasmon-enhanced nanomaterial technologies.

\section{EXPERIMENTAL SECTION}

\section{Synthesis of gold-silver core-shell nanoparticles}

The gold-silver core-shell nanoparticles are prepared following a modified procedure, which has been described previously. ${ }^{8,12}$ Gold chloride, sodium citrate, silver nitrate, L-ascorbic acid, potassium iodide, and sodium hydroxide are purchased from Sigma-Aldrich and used without further purification. The gold seed nanoparticles are first synthesized by adding $900 \mu \mathrm{l}$ of $34 \mathrm{mM}$ sodium citrate to $30 \mathrm{ml}$ of $290 \mu \mathrm{M}$ gold chloride in ultrapure water under boiling conditions with vigorous stirring. ${ }^{6,59,60}$ The solution undergoes a color change from pale yellow to bright red after $10 \mathrm{~min}$, resulting in the formation of $13.8 \pm 1.0 \mathrm{~nm}$ gold seed nanoparticles, as described in greater detail in the supplementary material. Gold-silver core-shell nanoparticles are prepared by reducing $\mathrm{Ag}^{+}$ onto the gold seed nanoparticles in solution using ultrapure water under three different seed concentrations. ${ }^{61}$ A volume of 17.5, 22.5, or $27.5 \mu \mathrm{l}$ of the prepared gold seed nanoparticle solution is added to an aqueous solution of $0.15 \mathrm{mM} \mathrm{AgNO}_{3}$ and $0.30 \mu \mathrm{M}$ of $\mathrm{KI}$ in a total volume of $2.5 \mathrm{ml}$. These constitute the three different reaction 
conditions, which result in the three different final gold-silver coreshell nanoparticle samples. A rapid addition of $5.0 \mu \mathrm{l}$ of $100 \mathrm{mM}$ ascorbic acid and $6.25 \mu \mathrm{l}$ of $100 \mathrm{mM} \mathrm{NaOH}$ initiates the silver shell growth at the colloidal surface of the gold seed nanoparticles. These three gold-silver core-shell nanoparticle reactions are all performed in a quartz cuvette under constant stirring while being monitored spectroscopically by in situ SHG and extinction spectroscopy, as described below.

\section{In situ second harmonic generation and extinction spectroscopy setup}

The experimental setup for in situ SHG spectroscopy consists of an ultrafast laser system, an optical setup, and a high-sensitivity charge-coupled device (CCD) spectroscopy detector connected to monochromator spectrograph, as described previously., 4,65 The output beam from a titanium:sapphire laser system with $2.7 \mathrm{~W}$ average power and $75 \mathrm{fs}$ pulses centered at $800 \mathrm{~nm}$ at a repetition rate of $80 \mathrm{MHz}$ is attenuated to $600 \mathrm{~mW}$ using a neutral density filter and is focused into a $1 \mathrm{~cm}$ by $1 \mathrm{~cm}$ quartz cuvette containing the colloidal nanoparticle sample under investigation. Optical filters are used to remove any residual SHG signal from the laser beam before the sample and to remove the fundamental $800 \mathrm{~nm}$ light after the sample while transmitting the SHG, which is collected in the forward direction and refocused into a spectroscopy detector. Additionally, a low-intensity broadband tungsten filament lamp is placed orthogonal to the path of the laser beam, collimated using a pair of lenses and focused into the quartz cuvette, using a fiberoptic spectrometer detector for the in situ extinction spectroscopy. For each trial, the gold seeds, $\mathrm{AgNO}_{3}$, and $\mathrm{KI}$ are added in ultrapure water for baseline measurements prior to the addition of AA and $\mathrm{NaOH}$, which initialize the reaction at time zero. A homebuilt data acquisition program collects 6 in situ SHG spectra and 6 background spectra using a computer-controlled beam block, followed by 10 in situ extinction spectra, in repeating iterations for time-dependent SHG and extinction spectroscopy with statistical analysis. The SHG acquisition times are $0.2 \mathrm{~s}$ for the first $50 \mathrm{~s}$, followed by $1 \mathrm{~s}$ for the remainder of each reaction, and the extinction acquisition time is $0.5 \mathrm{~s}$. A schematic diagram of the in situ SHG and extinction spectroscopy setup is shown in the supplementary material.

\section{Finite-difference time-domain calculations}

The extinction spectra of single gold-silver core-shell nanoparticles of various sizes in water are calculated using a classical finite-difference time-domain (FDTD) approach, as described previously. ${ }^{62}$ The FDTD approach provides solutions to Maxwell's equations for the plasmonic nanoparticle using discretized grids in space and time with spatial- and frequency-dependent permittivity and permeability fit to the bulk experimental values. ${ }^{62-64}$ For each calculation, the grid space is 20 a.u. $=1.06 \mathrm{~nm}$ and the time step is chosen to be 0.067 a.u. $=1.62 \times 10^{-3}$ fs such that the Courant-FriedrichsLewy stability condition equals to 0.8 . The total running time is set as 1500 a.u. $=36.3$ fs. The FDTD calculated extinction spectra allow for interpreting the observed experimental spectra features, as well as confirming the nanoparticle geometries. See the supplementary material for more details.

\section{RESULTS AND DISCUSSION}

After each gold-silver core-shell nanoparticle synthesis, the final nanoparticle size distribution is characterized by transmission electron microscopy using a survey of more than 200 nanoparticles for each sample. The resulting nanoparticle size histograms are fitted using log-normal distributions for each core-shell nanoparticle sample, as shown in the supplementary material. Figure 1 shows representative TEM images for the core-shell nanoparticle samples prepared using $27.5,22.5$, and $17.5 \mu \mathrm{l}$ of precursor gold seeds, resulting in final sizes of $51.0 \pm 7.1,65.8 \pm 9.2$, and $77.7 \pm 8.6 \mathrm{~nm}$, respectively, shown in order of increasing nanoparticle size. All gold-silver coreshell nanoparticles are observed to be relatively monodisperse and spherical in shape. By decreasing the amount of gold nanoparticles seeds added to the reaction, more $\mathrm{Ag}^{+}$is available per seed, resulting in larger silver shell thicknesses after the reaction is complete, in an analogous manner to previous work on seed-mediated gold nanoparticles.

The growth dynamics of each colloidal core-shell nanoparticle sample are monitored in real time by both in situ extinction spectroscopy and in situ SHG measurements. Representative extinction spectra for the gold-silver core-shell nanoparticle samples
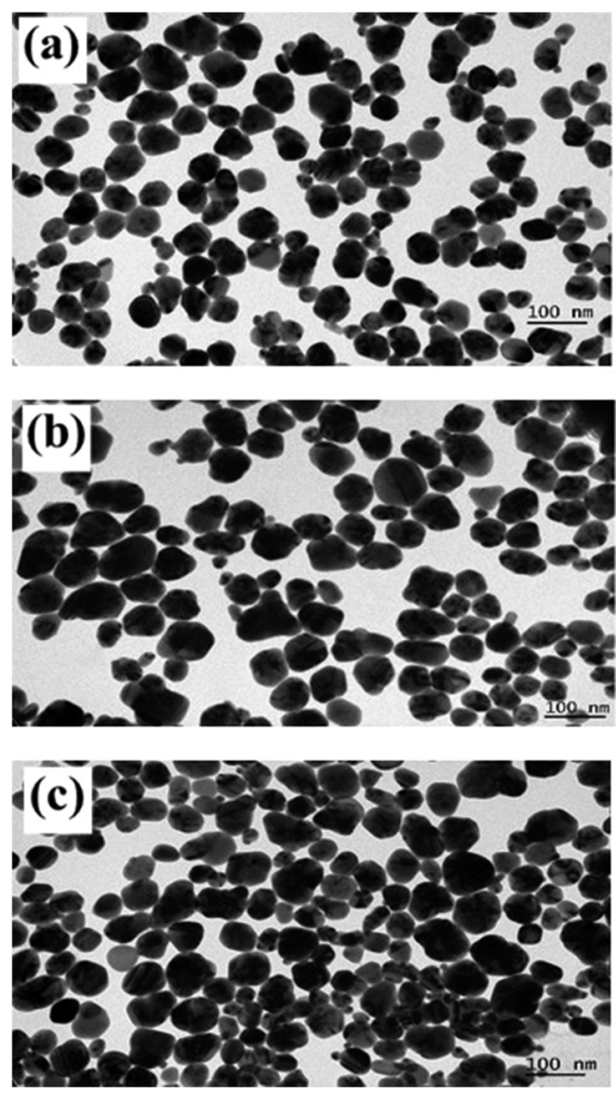

FIG. 1. Representative TEM images of gold-silver core-shell nanoparticles with average sizes of (a) $51.0 \pm 7.1$, (b) $65.8 \pm 9.2$, and (c) $77.7 \pm 8.6 \mathrm{~nm}$, prepared using $27.5,22.5$, and $17.5 \mu \mathrm{l}$ of precursor gold seeds, respectively. All Au@Ag nanoparticles have a gold core of diameter $13.8 \pm 1.0 \mathrm{~nm}$. 
using 27.5, 22.5, and $17.5 \mu \mathrm{l}$ of added gold precursor seeds at different reactions times are shown in Fig. 2. The initial extinction spectra correspond to the sample of gold seeds together with the $\mathrm{AgNO}_{3}$ and $\mathrm{KI}$ before to the addition of $\mathrm{AA}$ and $\mathrm{NaOH} .{ }^{57,65}$ Immediately after the addition of the reducing agent $\mathrm{AA}$, a broad plasmonic peak centered near $420 \mathrm{~nm}$ is observed. As the nanoparticle reaction continues in time, the plasmonic peak rapidly increases in intensity, while slightly blue shifting and narrowing in spectral width. After approximately $20 \mathrm{~s}$, all the gold-silver core-shell nanoparticle samples reach a stable configuration, and the extinction spectra are observed to remain constant over the rest of the in situ extinction measurements. The peak wavelengths of the final extinction spectra are $419 \mathrm{~nm}$, $424 \mathrm{~nm}$, and $428 \mathrm{~nm}$ for the $51 \mathrm{~nm}, 66 \mathrm{~nm}$, and $78 \mathrm{~nm} \mathrm{Au@Ag}$ samples, respectively, demonstrating a trend of a red-shifting
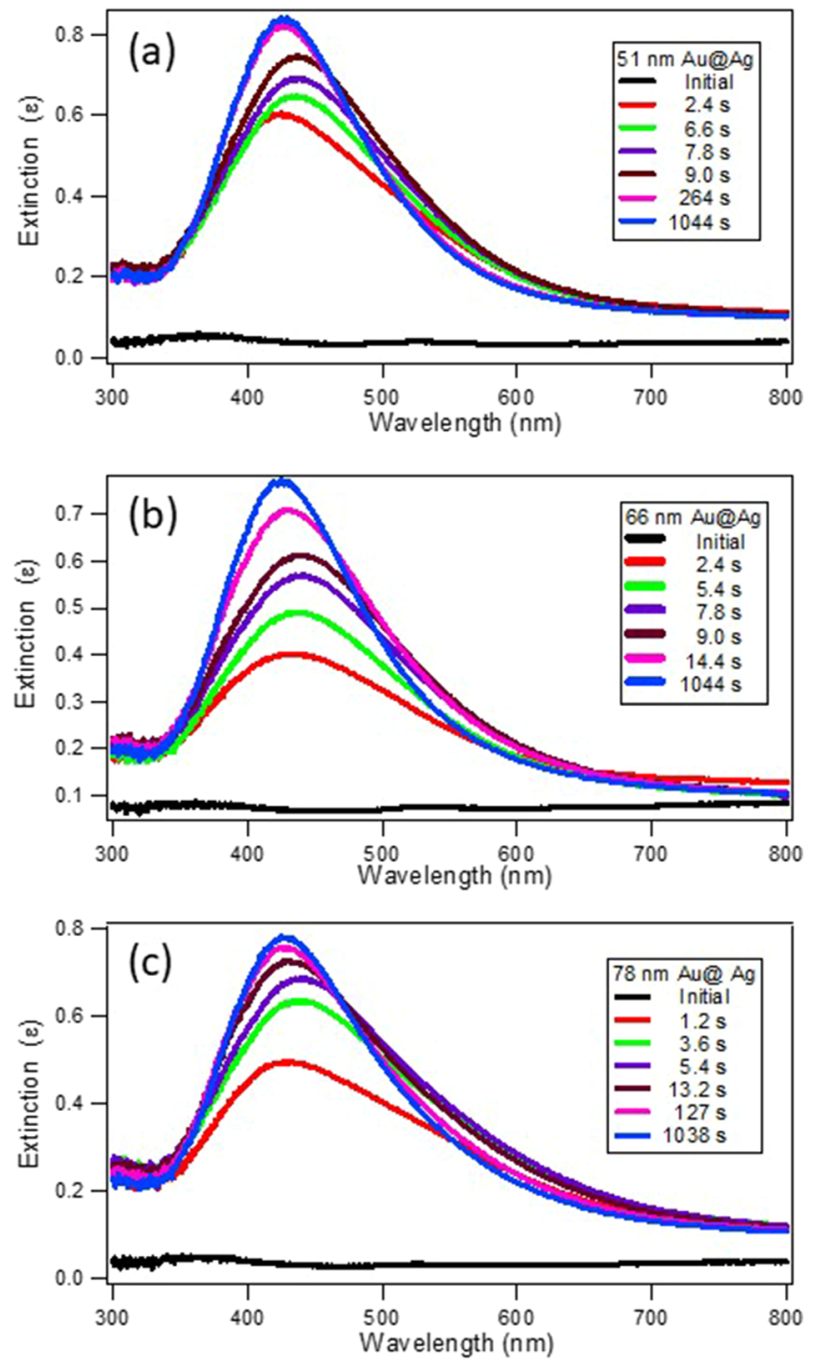

FIG. 2. In situ extinction spectra of gold-silver core-shell nanoparticles synthesized using (a) 27.5, (b) 22.5, and (c) $17.5 \mu \mathrm{l}$ of precursor gold seeds, respectively, at different times during the reactions. All Au@Ag nanoparticles have a gold core of diameter $13.8 \mathrm{~nm}$ plasmon peak as the silver shell increases in thickness. The full width at half maxima (FWHM) obtained from these final extinction spectra are $111 \mathrm{~nm}, 135 \mathrm{~nm}$, and $150 \mathrm{~nm}$ for the $51 \mathrm{~nm}, 66 \mathrm{~nm}$, and $78 \mathrm{~nm}$ Au@Ag samples, respectively, showing a trend of increasing spectral width as the silver shell thickness increases.

To better characterize the in situ extinction spectroscopy of the nanoparticle reaction dynamics, the extinction peak intensities are fit as a function of time for each core-shell nanoparticle sample. The extinction peak time traces are fit using an exponential function given by the equation $\varepsilon(t)=A e^{-t / \tau_{e x t}}+B$, where $t$ is the reaction time after addition of the reducing agent, $\tau_{\text {ext }}$ is the extinction growth lifetime, $A$ is the amplitude, and $B$ is the offset extinction value. The corresponding fits for each core-shell nanoparticle sample are shown in Fig. 3, and the fitting parameters are
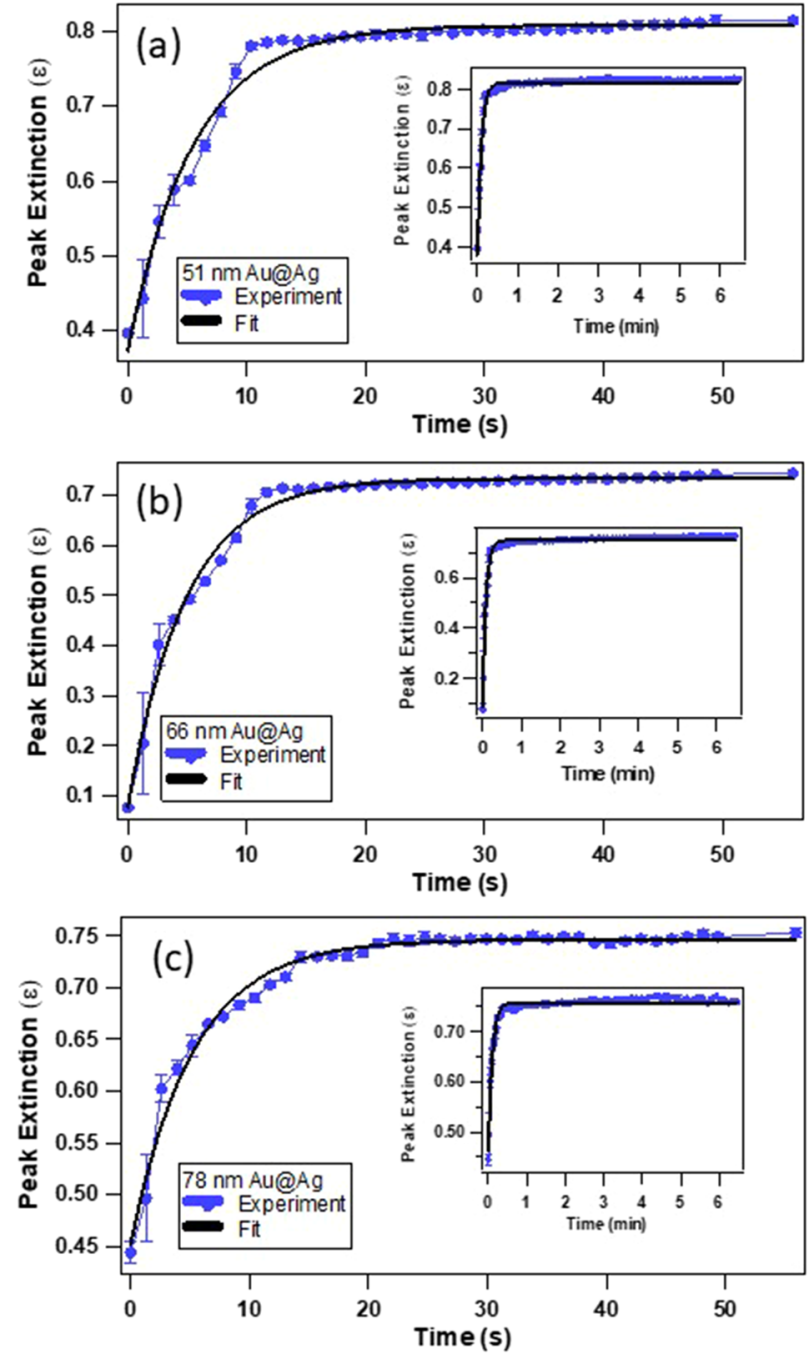

FIG. 3. Peak extinctions (blue circles) as a function of time for gold-silver core-shell nanoparticles using (a) 27.5, (b) 22.5, and (c) $17.5 \mu \mathrm{l}$ of precursor gold seeds, respectively, compared to the fits (black lines). 
tabulated in the supplementary material. The extinction growth lifetime increases as the final nanoparticle diameter increases with values of $5.2 \pm 0.2 \mathrm{~s}, 5.3 \pm 0.2 \mathrm{~s}$, and $6.2 \pm 0.1 \mathrm{~s}$ for the $51.0 \mathrm{~nm}$, $65.8 \mathrm{~nm}$, and $77.7 \mathrm{~nm}$ gold-silver core-shell nanoparticles, respectively. All Au@Ag nanoparticle samples investigated here have a $13.8 \mathrm{~nm}$ gold core, as determined by TEM measurements. These lifetimes can be best understood as the time it takes for the silver shells to grow around the gold seed nanoparticles to form the final gold-silver core-shell nanoparticle architectures. After the colloidal core-shell nanoparticle structures are formed according to these characteristic extinction growth lifetimes, the extinction spectra and intensities remain constant for the entire duration of the experiment, as shown in the insets of Fig. 3. These constant extinction spectra after the nanoparticle growth dynamics are complete indicate that the final core-shell nanoparticles remain very stable in colloidal suspension. Subsequent extinction spectroscopy measurements show that these core-shell nanoparticles are stable for at least several days in aqueous colloidal suspension after the nanoparticle synthesis is complete.

For additional analysis, the final extinction spectrum of each gold-silver core-shell nanoparticle sample is directly compared to the corresponding spectra generated using FDTD calculations. The extinction spectra for 9 different gold-silver core-shell nanoparticle sizes are calculating using FDTD, where each Au@Ag nanoparticle has a spherical gold core of diameter $13.8 \mathrm{~nm}$ and different spherical silver shell thicknesses. The 9 different silver shell thicknesses used for these calculations are 12.0, 13.7, 18.6, 23.0, 27.0, 29.0, 32.0, 35.0 , and $37.0 \mathrm{~nm}$, which result in total core-shell diameters ranging from $37.8 \mathrm{~nm}$ to $87.8 \mathrm{~nm}$. Additional details and results regarding the FDTD calculations of the gold-silver core-shell nanoparticle extinction spectra are provided in the supplementary material. The experimental extinction spectra are first compared with the normalized calculated spectra for single core-shell nanoparticles, using shell thicknesses of $18.6 \mathrm{~nm}, 27 \mathrm{~nm}$, and $32 \mathrm{~nm}$, to closely match the average sizes of the corresponding Au@Ag nanoparticles. The blue curves in Fig. 4 show the computed FDTD spectra for these nanoparticle geometries. In general, the spectra show trends of increasing peak intensities, red-shifted peak wavelengths, and increasing full widths at half maximum as the silver shell thickness increases, in general agreement with the experimental results. These calculated spectra from single Au@Ag nanoparticles have peak wavelengths of $410 \mathrm{~nm}, 424 \mathrm{~nm}$, and $449 \mathrm{~nm}$, showing a general agreement with the experimental results; however, the experimental extinction spectra show greater spectral widths and a lower degree of spectral red shifting than the single nanoparticle calculated spectra.

To analyze the influence of nanoparticle size distribution, the extinction spectra using a distribution of sizes are calculated, taking the five closest sizes for each Au@Ag nanoparticle sample with corresponding weighting to match the size distributions from the TEM measurements. These calculated spectra from distributions of Au@Ag nanoparticles, displayed as green curves in Fig. 4, show increased spectral widths compared with the corresponding single nanoparticles. However, the experimental spectra still have greater spectral widths and lower amounts of red shifting as compared to the calculated spectra. From these simulations, we conclude that polydispersity in the nanoparticle sizes only play a small role in the observed experimental spectral broadening. Instead, the
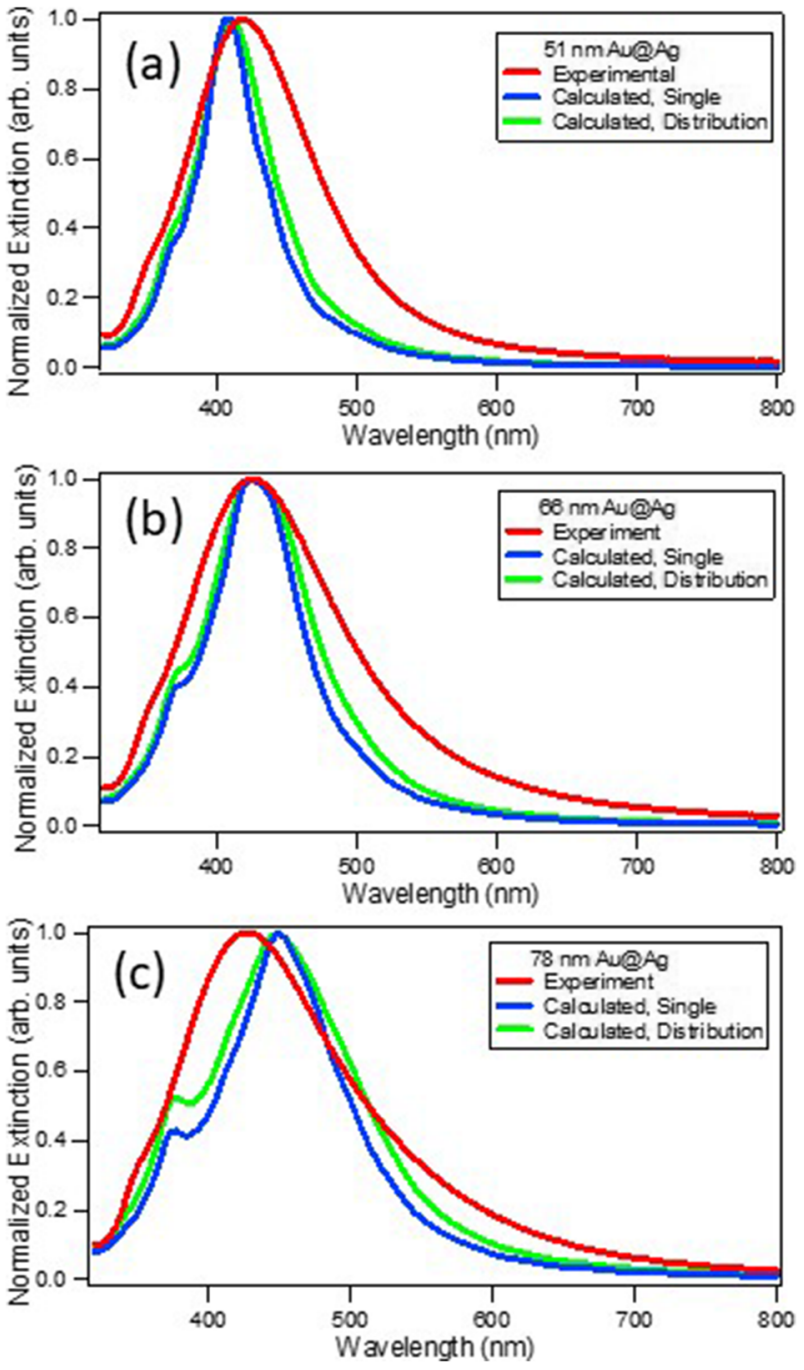

FIG. 4. Normalized final extinction spectra (red line) of gold-silver core-shell nanoparticles prepared using (a) 27.5, (b) 22.5, and (c) $17.5 \mu \mathrm{l}$ of precursor gold seeds, respectively, compared to corresponding spectra calculated using an FDTD approach from a single Au@Ag particle (blue line) and from a distribution of sizes (green line). The discrepancies between experiment and FDTD spectra are likely due to polydispersity in nanoparticle shapes and surface/interfacial roughness.

broadening is likely due to polydispersity in shape and surface morphology, as well as possible imperfections at the buried gold-silver core-shell interface.

In situ SHG spectroscopy provides a powerful and complementary tool to understand the nanoparticle growth dynamics, especially due to the surface sensitivity of the technique. ${ }^{34,35}$ Representative in situ SHG spectra for the Au@Ag sample prepared using $17.5 \mu \mathrm{l}$ of gold seeds at selected reaction times are shown in Fig. 5. The SHG signals, with peaks centered at $400 \mathrm{~nm}$ and with full widths at half maximum of $4.5 \mathrm{~nm}$, are clearly distinguished from the two-photon fluorescence signals at longer wavelengths. Additional in situ SHG 


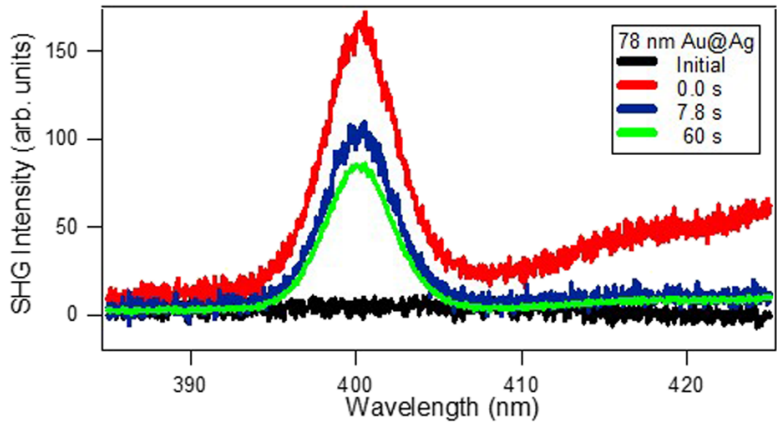

FIG. 5. SHG spectra for gold-silver core-shell nanoparticles prepared using 17.5 $\mu \mathrm{l}$ of precursor gold seeds at different times during the reaction. The lower level of noise in the SHG spectrum at $60 \mathrm{~s}$ is due to longer acquisition times.

spectra for the Au@Ag sample prepared using 22.5 and $27.5 \mu \mathrm{l}$ of gold seeds are shown in the supplementary material. The addition of $\mathrm{AA}$ and $\mathrm{NaOH}$ to the mixture of gold seeds, $\mathrm{AgNO}_{3}$, and $\mathrm{KI}$ is designated as time zero, initiating the nanoparticle growth dynamics of the silver shell around the gold core, as described previously. The lower level of noise in the last SHG spectrum is a consequence of five-times longer acquisition times. The time-dependent SHG electric field during the silver shell growth is shown in Fig. 6 for the three different gold-silver core-shell nanoparticle samples. The SHG signal is corrected to account for the time-dependent linear extinction response using the equation, $I_{S H G, \text { corr }}=I_{S H G} \exp \left(\varepsilon_{800}+\frac{1}{2} \varepsilon_{400}\right)$, where $I_{S H G}$, $\varepsilon_{800}$, and $\varepsilon_{400}$ represent the measured SHG intensity, the extinction at $800 \mathrm{~nm}$, and the extinction at $400 \mathrm{~nm}$, respectively. ${ }^{34}$ The time-dependent SHG electric field is calculated from the square root of the integrated SHG signal, where $E_{S H G}=\sqrt{I_{S H G, \text { corr }}}$. The SHG intensity before time zero is very low because the gold nanoparticle seeds are small in size and have a lower degree of resonance enhancement at the SHG wavelength. After the addition of the reducing agent AA to the solution, the SHG intensity increases rapidly, reaching a peak intensity, which is then followed by a biexponential decay as a function of time, where the relative intensities and corresponding lifetimes are different for each sample.

The SHG electric-field time traces are fit using a biexponential function given by $E_{S H G}(t)=A_{1} e^{-t / \tau_{1}}+A_{2} e^{-t / \tau_{2}}+B_{S H G}$, where $t$ is the reaction time after addition of the reducing agents, $\tau_{1}$ and $\tau_{2}$ are the associated growth lifetimes, $A_{1}$ and $A_{2}$ are the corresponding amplitudes, and $B_{S H G}$ is the offset SHG value. The corresponding fits are shown in Fig. 6, and fitting parameters are tabulated in the supplementary material. Here, $\tau_{1}$ and $\tau_{2}$ are denoted as the fast SHG lifetime and the slow SHG lifetime, respectively. The fast SHG lifetime $\tau_{1}$ increases as the final nanoparticle diameter increases with the values of $4.5 \pm 0.4 \mathrm{~s}, 6.5 \pm 0.3 \mathrm{~s}$, and $10.1 \pm 0.4 \mathrm{~s}$ for the final nanoparticle sizes of $51.0 \mathrm{~nm}, 65.8 \mathrm{~nm}$, and $77.7 \mathrm{~nm}$, respectively. Similarly, the slow SHG lifetime $\tau_{2}$ also increases as the final nanoparticle diameter increases with values of $170 \pm 8 \mathrm{~s}, 240 \pm 11 \mathrm{~s}$, and $264 \pm 17 \mathrm{~s}$ for the final nanoparticle sizes of $51.0 \mathrm{~nm}, 65.8 \mathrm{~nm}$, and $77.7 \mathrm{~nm}$, respectively. The final SHG electric field per nanoparticle can be estimated by dividing $B_{S H G}$ by the nanoparticle concentration, assuming each seed nanoparticle grows into a core-shell nanoparticle. This gives
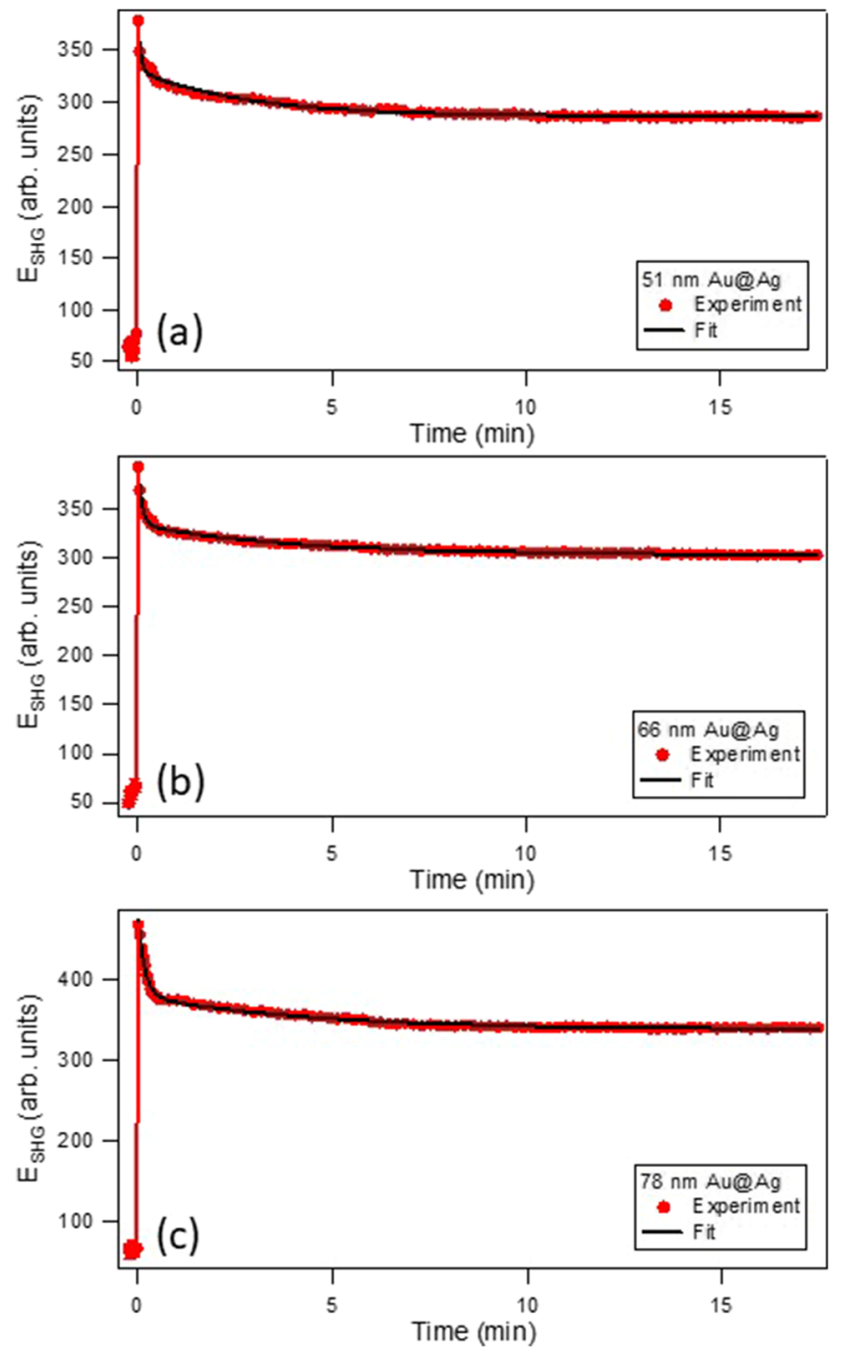

FIG. 6. SHG electric field (red circles) as a function of reaction time during the gold-silver core-shell nanoparticle reaction using (a) 27.5, (b) 22.5, and (c) $17.5 \mu \mathrm{l}$ of precursor gold seeds, compared to the corresponding fits (black lines).

an estimated final SHG electric field measured per nanoparticle of $(2.4 \pm 0.2) \times 10^{-8},(3.0 \pm 0.02) \times 10^{-8}$, and $(4.4 \pm 0.3) \times 10^{-8}$ for the $51 \mathrm{~nm}, 66 \mathrm{~nm}$, and $78 \mathrm{~nm} \mathrm{Au@Ag} \mathrm{nanoparticles,} \mathrm{respectively,} \mathrm{in}$ arbitrary units, showing increasing SHG signal as the nanoparticle size increases, in agreement with previous SHG studies of different types of nanoparticles. ${ }^{35,40,65,66}$ In addition, the SHG intensities of the Au@Ag nanoparticles have large plasmonic resonance enhancement at the second harmonic frequency.

A comparison of these SHG lifetimes with the corresponding extinction growth lifetimes provides insight into the silver shell growth dynamics. The extinction growth lifetime $\tau_{\text {ext }}$ as a function of the final nanoparticle diameter is shown in Fig. 7(a). The fast SHG lifetime $\tau_{1}$ and the slow SHG lifetime $\tau_{2}$ are plotted as a function of the final nanoparticle diameter in Figs. 7(b) and 7(c), respectively. The fast SHG lifetimes are approximately equal to the corresponding 

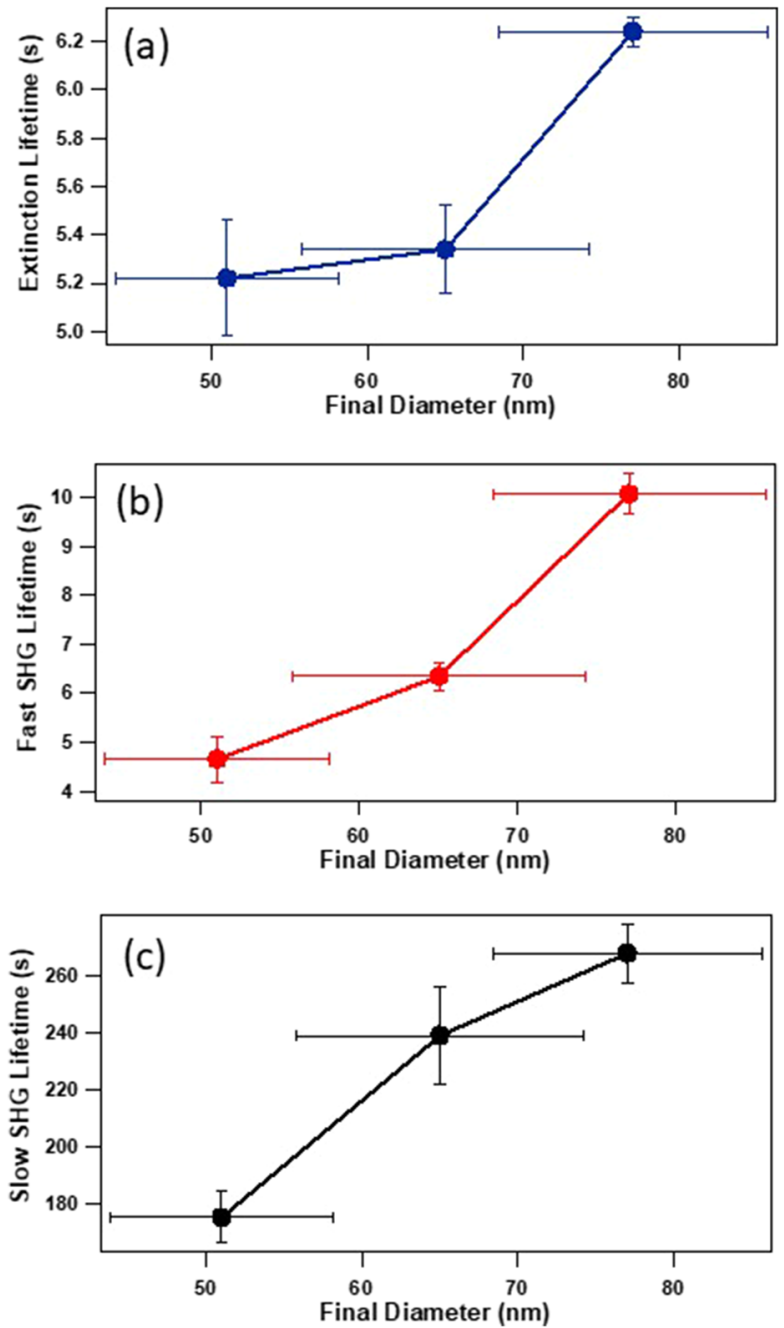

FIG. 7. The measured (a) extinction growth lifetime, (b) fast SHG lifetime, and (c) slow SHG lifetime as a function of final core-shell nanoparticle diameter.

extinction growth lifetimes, so $\tau_{1}$ is also attributed to the timescale of the silver shell growth, where slight deviations between $\tau_{1}$ and $\tau_{\text {ext }}$ are caused by the different associated optical processes, with $\tau_{1}$ having enhanced surface sensitivity. However, the slow SHG lifetimes $\tau_{2}$ occurs over much longer timescales, with the SHG signal continuing to decrease significantly even as the extinction spectrum and intensity remain constant. Therefore, the slow SHG lifetime $\tau_{2}$ is attributed to changes in the nanoparticle surface charge density caused by the $\chi^{(3)}$ effect. ${ }^{4,36,37}$ For additional analysis, zeta potential measurements are taken for the $66 \mathrm{~nm}$ gold-silver core-shell nanoparticles as a function of time after the addition of the reducing agents, as shown in Fig. S10 in the supplementary material. The zeta potential is shown to vary on the same timescale as the slow SHG lifetime, becoming less negative over time, in agreement with corresponding surface charge density changes resulting in an SHG $\chi^{(3)}$ effect. Ascorbic acid plays a major role as the reducing agent of
$\mathrm{Ag}^{+}$at the nanoparticle surface, and it can exist as both a carboxylic acid and an ascorbate anion conjugate base in various forms, with $\mathrm{pH}$-dependent reactivity. ${ }^{8,12,68,69}$ As the reaction proceeds, ascorbic acid is converted to dehydroascorbic acid for the reduction of two $\mathrm{Ag}^{+}$ions, releasing two protons. ${ }^{68}$ Excess ascorbate anions at the surface can then react back to ascorbic acid or leave the surface, resulting in a decreased surface charge density and a decreased SHG signal from the $\chi^{(3)}$ term. However, more work is needed to fully characterize the nanoparticle surface chemistry and the corresponding surface charge density during the nanoparticle shell growth reaction.

By analyzing both the in situ SHG and extinction spectroscopy together, with comparison to the FDTD calculations, a more extensive understanding of the gold-silver core-shell growth process is obtained. The results of the in situ SHG and extinction measurements of the Au@Ag nanoparticle growth dynamics can be described using a three-step model. The sudden rise in SHG signal immediately after the addition of AA constitutes the first step which is attributed to a period of rapid growth in shell size where the nanoparticle surface has a rough, uneven morphology characterized by nanoclustered domains or porous substructures. These bumpy surfaces generate increased SHG intensities due to plasmonic hot spots, similar to previous in situ SHG measurements from gold nanoparticle growth reactions. ${ }^{34,35}$ This first step occurs on a timescale that is faster than our current experimental temporal resolution. In the second step, the silver shell continues to grow and the nanoparticle surface becomes smoother over time, where the decrease in plasmonic hot spots causes the overall SHG signal to decrease. This second step has characteristic lifetimes corresponding to $\tau_{1}$ and $\tau_{\text {ext }}$, where the in situ extinction spectra blue shifts and narrows over time, consistent with this picture, ${ }^{35,70}$ and where the shell growth lifetime increases with increasing nanoparticle size, in agreement with similar studies. ${ }^{35,71}$ Finally, in the third step, the overall Au@Ag nanoparticle size remains constant and the corresponding extinction spectrum also remains constant, but additional nanoparticle surface chemistry reactions continue such that the surface charge density decreases causing the SHG signal to decrease from the $\chi^{(3)}$ effect on timescales of $\tau_{2}$. Together, in situ SHG and extinction spectroscopy provides complementary information to study the dynamics of different processes involved in size-dependent gold-silver core-shell nanoparticle growth reactions in solution for potential advances in nanoscale engineering.

\section{CONCLUSION}

In situ second harmonic generation and extinction spectroscopy are used to investigate the size-dependent growth dynamics of gold-silver core-shell nanoparticles in water. The in situ extinction spectra show rapid peak intensity increases upon the addition of ascorbic acid as the reducing agent to solutions of $14 \mathrm{~nm}$ colloidal gold seed nanoparticles and silver cations, with characteristic silver shell growth lifetimes of approximately 5-6 s. The nanoparticle growth reactions result in stable core-shell nanoparticles that vary in size from approximately 51 to $78 \mathrm{~nm}$ in diameter, depending on the initial seed concentration. The in situ SHG results show an abrupt signal increase followed by a biexponential decrease, where the faster SHG lifetimes are on the same timescale as the changes 
in extinction spectroscopy, corresponding to the shell growth process, while the slower SHG lifetimes of approximately 3-5 min are attributed to changes in the nanoparticle surface charge density. The final extinction spectra are compared with finite-difference timedomain calculations, displaying general agreement, where the plasmon peak increases in intensity, red shifts, and increases in spectral width as the silver shell increases in thickness. Based on the FDTD results, the broader experimental spectra are likely caused by distributions in nonspherical morphologies and surface/interfacial roughness, as opposed to polydispersity in size. The overall core-shell nanoparticle growth reaction is consistent with a three-step model, where the silver shell first grows abruptly with a large degree of plasmonic hot spots due to a rough, uneven surface, followed by continued growth with accompanying surface smoothening, followed by a final step where the nanoparticle core-shell structure remains fixed in size but surface reactions continue, which decrease the surface charge density leading to the final, stable colloidal Au@Ag nanoparticle sample. These in situ SHG and extinction spectroscopy results, combined with FDTD calculations, provide a sensitive method for studying the real-time growth dynamics of colloidal gold-silver coreshell nanoparticles and similar hybrid core-shell structures that can be utilized in developing advanced plasmonic nanomaterial applications.

\section{SUPPLEMENTAL MATERIAL}

See the supplementary material for additional details on the nanoparticle characterization measurements, experimental setup, and FTDT calculations.

\section{ACKNOWLEDGMENTS}

Generous financial support for this work was provided by Louisiana State University. L.H.H., J.C.R., A.S.D., P.H., and R.A.K. gratefully acknowledge financial support from the National Science Foundation EPSCoR CIMM project under Award No. OIA1541079. M.C. and K.L. gratefully acknowledge support by the U.S. Department of Energy, Office of Science, Basic Energy Sciences, Early Career Program, under Award No. DE-SC0017868. The authors also thank the LSU Material Characterization Center (LSUMCC) and Ying Xiao for assistance with transmission electron microscopy.

\section{REFERENCES}

${ }^{1}$ A. J. Haes and R. P. Van Duyne, J. Am. Chem. Soc. 124(35), 10596-10604 (2002). ${ }^{2}$ R. A. Sperling, P. R. Gil, F. Zhang, M. Zanella, and W. J. Parak, Chem. Soc. Rev. 37(9), 1896-1908 (2008).

${ }^{3}$ X. Huang, I. H. El-Sayed, W. Qian, and M. A. El-Sayed, J. Am. Chem. Soc. 128(6), $2115-2120$ (2006)

${ }^{4}$ R. R. Kumal, T. E. Karam, and L. H. Haber, J. Phys. Chem. C 119(28), 16200-16207 (2015)

${ }^{5}$ P. V. Kamat, J. Phys. Chem. C 111(7), 2834-2860 (2007)

${ }^{6}$ T. E. Karam and L. H. Haber, J. Phys. Chem. C 118(1), 642-649 (2013).

${ }^{7}$ R. R. Kumal, C. R. Landry, M. Abu-Laban, D. J. Hayes, and L. H. Haber, Langmuir 31(36), 9983-9990 (2015).

${ }^{8}$ R. R. Kumal, M. Abu-Laban, P. Hamal, B. Kruger, H. T. Smith, D. J. Hayes, and L. H. Haber, J. Phys. Chem. C 122(34), 19699-19704 (2018).

${ }^{9}$ S. Link and M. A. El-Sayed, Int. Rev. Phys. Chem. 19(3), 409-453 (2000).

${ }^{10}$ I. H. El-Sayed, X. Huang, and M. A. El-Sayed, Nano Lett. 5(5), 829-834 (2005).
${ }^{11}$ A. E. Prigodich, O.-S. Lee, W. L. Daniel, D. S. Seferos, G. C. Schatz, and C. A. Mirkin, J. Am. Chem. Soc. 132(31), 10638-10641 (2010).

${ }^{12}$ T. E. Karam, H. T. Smith, and L. H. Haber, J. Phys. Chem. C 119(32), 18573-18580 (2015).

${ }^{13}$ R. Ho-Wu, P. K. Sahu, N. Wu, T. Chen, C. Yu, J. Xie, and T. Goodson III, J. Phys. Chem. C 122, 24368-24379 (2018).

${ }^{14}$ S. C. Abeyweera, K. D. Rasamani, and Y. Sun, Acc. Chem. Res. 50(7), 1754-1761 (2017).

${ }^{15}$ T. Zhao, M. Steves, B. S. Chapman, J. B. Tracy, and K. L. Knappenberger, Anal. Chem. 90, 13702-13707 (2018).

${ }^{16}$ C. J. DeSantis, R. G. Weiner, A. Radmilovic, M. M. Bower, and S. E. Skrabalak, J. Phys. Chem. Lett. 4(18), 3072-3082 (2013).

${ }^{17}$ R. G. Weiner, M. R. Kunz, and S. E. Skrabalak, Acc. Chem. Res. 48(10), 2688-2695 (2015).

${ }^{18}$ K. D. Gilroy, A. Ruditskiy, H.-C. Peng, D. Qin, and Y. Xia, Chem. Rev. 116(18), 10414-10472 (2016).

${ }^{19}$ X. Ye, H. Shi, X. He, K. Wang, D. Li, and P. Qiu, J. Mater. Chem. B 2(23), 3667-3673 (2014).

${ }^{20}$ E. T. Vickers, M. Garai, S. B. Naghadeh, S. Lindley, J. Hibbs, Q.-H. Xu, and J. Z. Zhang, J. Phys. Chem. C 122(25), 13304-13313 (2017).

${ }^{21}$ S. A. Lindley and J. Z. Zhang, ACS Appl. Nano Mater. 2(2), 1072-1081 (2019).

${ }^{22}$ H. F. Zarick, A. Boulesbaa, E. M. Talbert, A. Puretzky, D. Geohegan, and R. Bardhan, J. Phys. Chem. C 121(8), 4540-4547 (2017).

${ }^{23}$ L. Lu, H. Wang, Y. Zhou, S. Xi, H. Zhang, J. Hu, and B. Zhao, Chem. Commun. 38(2), 144-145 (2002).

${ }^{24}$ C. B. Norris, P. R. Joseph, M. R. Mackiewicz, and S. M. Reed, Chem. Mater. 22(12), 3637-3645 (2010).

${ }^{25}$ L. Lu, G. Burkey, I. Halaciuga, and D. V. Goia, J. Colloid Interface Sci. 392, 90-95 (2013).

${ }^{26}$ M. Tsuji, N. Miyamae, S. Lim, K. Kimura, X. Zhang, S. Hikino, and M. Nishio, Cryst. Growth Des. 6(8), 1801-1807 (2006).

${ }^{27}$ C. M. Gonzalez, Y. Liu, and J. Scaiano, J. Phys. Chem. C 113(27), 11861-11867 (2009).

${ }^{28}$ K. L. McGilvray, C. Fasciani, C. J. Bueno-Alejo, R. Schwartz-Narbonne, and J. C. Scaiano, Langmuir 28(46), 16148-16155 (2012).

${ }^{29}$ S. Anandan, F. Grieser, and M. Ashokkumar, J. Phys. Chem. C 112(39), 15102-15105 (2008).

${ }^{30}$ R. G. Chaudhuri and S. Paria, Chem. Rev. 112(4), 2373-2433 (2011).

${ }^{31}$ T. J. Woehl, J. E. Evans, I. Arslan, W. D. Ristenpart, and N. D. Browning, ACS Nano 6(10), 8599-8610 (2012).

${ }^{32}$ S. F. Tan, S. W. Chee, G. Lin, M. Bosman, M. Lin, U. Mirsaidov, and C. A. Nijhuis, J. Am. Chem. Soc. 138(16), 5190-5193 (2016).

${ }^{33}$ J. Polte, X. Tuaev, M. Wuithschick, A. Fischer, A. F. Thuenemann, K. Rademann, R. Kraehnert, and F. Emmerling, ACS Nano 6(7), 5791-5802 (2012).

${ }^{34}$ C. Sauerbeck, M. Haderlein, B. Schürer, B. Braunschweig, W. Peukert, and R. N. K. Taylor, ACS Nano 8(3), 3088-3096 (2014).

${ }^{35}$ R. A. Khoury, J. C. Ranasinghe, A. S. Dikkumbura, P. Hamal, R. R. Kumal, T. E. Karam, H. T. Smith, and L. H. Haber, J. Phys. Chem. C 122(42), 24400-24406 (2018).

${ }^{36}$ P. L. Hayes, J. N. Malin, D. S. Jordan, and F. M. Geiger, Chem. Phys. Lett. 499(4-6), 183-192 (2010)

${ }^{37}$ E. C. Yan, Y. Liu, and K. B. Eisenthal, J. Phys. Chem. B 102(33), 6331-6336 (1998).

${ }^{38}$ K. B. Eisenthal, Chem. Rev. 106(4), 1462-1477 (2006).

${ }^{39}$ Y. Shen, Nature 337(6207), 519 (1989).

${ }^{40}$ S.-H. Jen, G. Gonella, and H.-L. Dai, J. Phys. Chem. A 113(16), 4758-4762 (2009).

${ }^{41}$ Z. Song, S. Sarkar, A. D. Vogt, G. D. Danzer, C. J. Smith, E. J. Gualtieri, and G. J. Simpson, Anal. Chem. 90(7), 4406-4413 (2018).

${ }^{42}$ P. E. Ohno, S. A. Saslow, H.-f. Wang, F. M. Geiger, and K. B. Eisenthal, Nat. Commun. 7, 13587 (2016).

${ }^{43}$ P. E. Ohno, H. Chang, A. P. Spencer, Y. Liu, M. D. Boamah, H.-f. Wang, and F. M. Geiger, J. Phys. Chem. Lett. 10, 2328-2334 (2019). 
${ }^{44}$ G. Gonella, C. Lütgebaucks, A. G. De Beer, and S. Roke, J. Phys. Chem. C 120(17), 9165-9173 (2016).

${ }^{45}$ R. R. Kumal, H. Nguyenhuu, J. E. Winter, R. L. McCarley, and L. H. Haber, J. Phys. Chem. C 121(29), 15851-15860 (2017).

${ }^{46}$ T. T. Nguyen, K. L. Sly, and J. C. Conboy, Anal. Chem. 84(1), 201-208 (2011).

${ }^{47}$ A. D. Fearon and G. Y. Stokes, J. Phys. Chem. B 121(46), 10508-10518 (2017).

${ }^{48}$ J. Liu, M. Subir, K. Nguyen, and K. B. Eisenthal, J. Phys. Chem. B 112(48), 15263-15266 (2008).

${ }^{49}$ A. M. Darlington and J. M. Gibbs-Davis, J. Phys. Chem. C 119(29), 16560-16567 (2015)

${ }^{50}$ L. B. Dreier, C. Bernhard, G. Gonella, E. H. Backus, and M. Bonn, J. Phys. Chem. Lett. 9(19), 5685-5691 (2018).

${ }^{51}$ Y. Liu, J. Dadap, D. Zimdars, and K. B. Eisenthal, J. Phys. Chem. B 103(13), 2480-2486 (1999).

${ }^{52}$ R. Jin, J. E. Jureller, H. Y. Kim, and N. F. Scherer, J. Am. Chem. Soc. 127(36), 12482-12483 (2005).

${ }^{53}$ F. W. Vance, B. I. Lemon, and J. T. Hupp, J. Phys. Chem. B 102(50), 10091-10093 (1998).

${ }^{54}$ E. Hao, G. Schatz, R. Johnson, and J. Hupp, J. Chem. Phys. 117(13), 5963-5966 (2002).

${ }^{55}$ I. Russier-Antoine, E. Benichou, G. Bachelier, C. Jonin, and P. Brevet, J. Phys. Chem. C 111(26), 9044-9048 (2007).

${ }^{56} \mathrm{G}$. Bachelier, J. Butet, I. Russier-Antoine, C. Jonin, E. Benichou, and P.-F. Brevet, Phys. Rev. B 82(23), 235403 (2010).

${ }^{57}$ L. H. Haber, S. J. Kwok, M. Semeraro, and K. B. Eisenthal, Chem. Phys. Lett. 507(1-3), 11-14 (2011).
${ }^{58}$ W. Gan, G. Gonella, M. Zhang, and H.-L. Dai, J. Chem. Phys. 134, 041104 (2011).

${ }^{59}$ S. D. Perrault and W. C. Chan, J. Am. Chem. Soc. 131(47), 17042-17043 (2009).

${ }^{60}$ K. R. Brown, D. G. Walter, and M. J. Natan, Chem. Mater. 12(2), 306-313 (2000).

${ }^{61}$ A. K. Samal, L. Polavarapu, S. Rodal-Cedeira, L. M. Liz-Marzán, J. Pérez-Juste, and I. Pastoriza-Santos, Langmuir 29(48), 15076-15082 (2013).

${ }^{62}$ H. T. Smith, T. E. Karam, L. H. Haber, and K. Lopata, J. Phys. Chem. C 121(31), 16932-16942 (2017).

${ }^{63}$ A. Coomar, C. Arntsen, K. A. Lopata, S. Pistinner, and D. Neuhauser, J. Chem. Phys. 135(8), 084121 (2011).

${ }^{64}$ K. Lopata and D. Neuhauser, J. Chem. Phys. 130(10), 104707 (2009).

${ }^{65}$ S. Roke and G. Gonella, Annu. Rev. Phys. Chem. 63, 353-378 (2012).

${ }^{66}$ J. I. Dadap, J. Shan, K. B. Eisenthal, and T. F. Heinz, Phys. Rev. Lett. 83(20), 4045 (1999).

${ }^{67}$ J.-P. Abid, J. Nappa, H. H. Girault, and P.-F. Brevet, J. Chem. Phys. 121(24), 12577-12582 (2004).

${ }^{68}$ S. Patra, D. Sen, A. K. Pandey, J. Bahadur, S. Mazumder, S. V. Ramagiri, J. R. Bellare, S. V. Roth, G. Santoro, and S. Yu, RSC Adv. 4(103), 59379-59386 (2014).

${ }^{69}$ Y. Qin, X. Ji, J. Jing, H. Liu, H. Wu, and W. Yang, Colloids Surf., A 372(1-3), 172-176 (2010).

${ }^{70}$ Y. Wang, P. H. Camargo, S. E. Skrabalak, H. Gu, and Y. Xia, Langmuir 24(20), 12042-12046 (2008).

${ }^{71}$ N. R. Jana, L. Gearheart, and C. J. Murphy, Chem. Mater. 13(7), 2313-2322 (2001). 